INPLASY

PROTOCOL

To cite: Zhang et al. Efficacy of Wen-Dan Decoction in the treatment of patients with coronary heart disease: $A$ protocol for systematic review and meta-analysis. Inplasy protocol 2021110001. doi: 10.37766/inplasy2021.11.0001

Received: 01 November 2021

Published: 01 November 2021

Corresponding author:

Yingwei Wang

13836113901@163.com

Author Affiliation:

Heilongjiang University of traditional Chinese Medicine.

Support: No financial support.

Review Stage at time of this submission: Preliminary searches.

Conflicts of interest: None declared.

\section{Efficacy of Wen-Dan Decoction in the treatment of patients with coronary heart disease: A protocol for systematic review and meta-analysis}

Zhang, XX'; Wang, YW2; Liu, LF3; Jiang, H4; Wang, J5; Xiao, Y6; Wang, JW7.

Review question / Objective: To evaluate the efficacy of WDD in the treatment of patients with CHD. And provide the latest evidence of evidence-based medicine for the clinical treatment of CHD.

Condition being studied: Coronary Heart Disease (CHD) is a heart disease caused by myocardial ischemia, hypoxia or necrosis due to stenosis or occlusion of lumen caused by coronary atherosclerosis. It belongs to ischemic cardiomyopathy and is more common in clinic. Previous studies have shown that Wen-Dan Decoction (WDD) is safe and effective, but there is a lack of systematic reviews. The purpose of this study is to systematically study the efficacy of WDD in the treatment of patients with CHD.

INPLASY registration number: This protocol was registered with the International Platform of Registered Systematic Review and Meta-Analysis Protocols (INPLASY) on 01 November 2021 and was last updated on 01 November 2021 (registration number INPLASY2021110001).

\section{INTRODUCTION}

Review question / Objective: To evaluate the efficacy of WDD in the treatment of patients with CHD. And provide the latest evidence of evidence-based medicine for the clinical treatment of CHD.

Condition being studied: Coronary Heart Disease (CHD) is a heart disease caused by myocardial ischemia, hypoxia or necrosis 
due to stenosis or occlusion of lumen caused by coronary atherosclerosis. It belongs to ischemic cardiomyopathy and is more common in clinic. Previous studies have shown that Wen-Dan Decoction (WDD) is safe and effective, but there is a lack of systematic reviews. The purpose of this study is to systematically study the efficacy of WDD in the treatment of patients with CHD.

\section{METHODS}

Participant or population: Refer to the International Society of Cardiology and Association and the World Health Organization Clinical Nomenclature Standardization Joint Task Group report "Nomenclature and Diagnostic Criteria of Ischemic Heart Disease" for patients diagnosed with CHD.

Intervention: The treatment of the experimental group was treated with WDD on the basis of conventional drug treatment. The mode of administration is oral, and the dosage form is decoction or granule.

Comparator: The control group received conventional medication or placebo treatment.

Study designs to be included: 1. Types of studiesAll randomized controlled trials of WDD in the treatment of CHD, whether blinded or unblinded.2. Type of participantsRefer to the International Society of Cardiology and Association and the World Health Organization Clinical Nomenclature Standardization Joint Task Group report "Nomenclature and Diagnostic Criteria of Ischemic Heart Disease" for patients diagnosed with CHD.3. Type of interventions. The treatment of the experimental group was treated with WDD on the basis of conventional drug treatment. The mode of administration is oral, and the dosage.

Eligibility criteria: (1) Non-RCT literature.(2) Animal experiments, case reports and reviews.(3) Duplicate literature.(4) Patients with UC and bacterial infection.(5) Articles for which data cannot be obtained.

Information sources: We will use 8 databases to search all relevant literature resources. Including four English databases PubMed, EMBASE, Web of Science, Central, and four Chinese databases China Biomedical Literature (СВM), the Wanfang Chinese digital periodical and conference database (WanFang Data), China National Knowledge Infrastructure database (CNKI), and the VIP Chinese Science and Technique Journals Database (VIP). The retrieval time starts from them until August 2021. Key words include "coronary heart disease", "wendan decoction" and "random allocation". We will also search for ongoing or unpublished trials from the National Institutes of Health (NIH) clinical registration clinical trials, the international clinical trial registration platform (ICTRP) and the China clinical trial registration platform.

Main outcome(s): The main outcome indicator is total effective rate. Secondary outcome indicators include symptom score, Nitroglycerin reduction rate. Efficacy evaluation of electrocardiogram etc.

Quality assessment / Risk of bias analysis: To assess the bias of the included literature, we will use the Cochrane risk assessment tool. Its main content includes the following 7 items: random method selection; allocation hiding; blind method, completeness of the result data; whether the evaluator is blind; selectively reporting results; other biases. The above 7 items all contain 3 options of "yes", "no" and "unclear", and are properly evaluated by 2 researchers. If there is a disagreement during the evaluation process, find a third party for discussion.

Strategy of data synthesis: RevMan V.5.3 software will be used for data analysis and quantitative data synthesis. For continuous data, the standard mean difference (SMD) with $95 \%$ confidence interval $(\mathrm{Cl})$ will be used for analysis. For dichotomous data, a 
risk ratio (RR) with $95 \% \mathrm{Cl}$ will be used for evaluation.

Subgroup analysis: If necessary, we will conduct a subgroup analysis based on the type of acupuncture, acupoints and other factors. In addition, we will also perform sensitivity analysis to test the stability of the results.

Sensitivity analysis: The 12 test statistic will be used to assess the heterogeneity of the included RCTs. If 120.1 , the results will indicate that there is no homogeneity in the study, and the fixed effects model will be used for analysis. Otherwise, it indicates that there is heterogeneity, and the reasons for the heterogeneity need to be further analyzed.

Language: Chinese and English.

Country(ies) involved: China.

Keywords: systematic review, coronary heart disease, Wendan Decoction.

Contributions of each author:

Author 1 - Xiaoyu Zhang.

Email: 1721896449@qq.com

Author 2 - Yingwei Wang.

Email: 13836113901@163.com

Author 3 - Lufei Liu.

Email: 8313781@qq.com

Author 4 - Hui Jiang.

Email: 1459840209@qq.com

Author 5 - Jing Wang.

EMail: 1663779277@qq.com

Author 6 - Yang Xiao.

Email: 1015286412@qq.com

Author 7 - Jianwei Wang.

Email: hawjw@163.com 